\title{
Clinical study of video-assisted thoracoscopic surgery wedge resection in early-stage lung cancer by tumor mapping with indocyanine green
}

\author{
Liang Zhong ${ }^{1}$, Weidong $\mathrm{Hu}^{2}$, Shuping $\mathrm{Li}^{3}$, Zhenhong Wei ${ }^{4}$, Zijiang $\mathrm{Zhu}^{1}$, Gang Jin ${ }^{1}$, Hongyi Zhang ${ }^{1}$, Yao Pang ${ }^{1}$, Jun Yu \\ ${ }^{1}$ Department of Thoracic Surgery, Gansu Province People's Hospital, Lanzhou, China \\ 2Department of Respiration, Gansu Province People's Hospital, Lanzhou, China \\ ${ }^{3}$ Department of Radiotherapy, Gansu Province People's Hospital, Lanzhou, China \\ ${ }^{4}$ Department of Clinical Laboratory, Gansu Province People’s Hospital, Lanzhou, China
}

Videosurgery Miniinv 2019; 14 (4): 545-550

DOI: https://doi.org/10.5114/wiitm.2019.89986

\begin{abstract}
Aim: This study aimed to assess the clinical effectiveness of video-assisted thoracoscopic surgery (VATS) in early-stage lung cancer by indocyanine green (ICG) for tumor mapping.

Material and methods: Thirty patients with early-stage lung cancer with peripheral nodules smaller than $2 \mathrm{~cm}$ scheduled for computed tomography (CT)-guided microcoil placement followed by ICG tumor mapping by VATS wedge resection were enrolled. After microcoil deployment, 100 to $150 \mathrm{ml}$ of diluted ICG was injected percutaneously near the nodule. The nodule initially was localized solely by using a near-infrared ray (NIR) thoracoscope to visualize ICG fluorescence. Thoracoscopic instruments were used to determine the staple line. Wedge resection was performed after confirmation of the location of the microcoil using fluoroscopy and pathology results.

Results: Thirty patients underwent VATS resection. The median tumor size was $1.3 \mathrm{~cm}$ by CT. The median depth from the pleural surface was $1.7 \mathrm{~cm}$ (range: $0.5-3.8 \mathrm{~cm}$ ). The median CT-guided intervention time was $25 \mathrm{~min}$, and VATS procedural time was $50 \mathrm{~min}$. ICG fluorescence was clearly identified in 30 of 30 patients (100\%). The surgical margins were all negative on final pathology in all included cases. The final diagnoses were 30 primary lung cancers; none needed additional resection.

Conclusions: CT-guided percutaneous ICG injection and intraoperative NIR localization of small nodules are safe and feasible. These offer surgeons the ease of localization through direct indocyanine green fluorescence imaging without the use of fluoroscopy and may be a complementary technique to preoperative microcoil placement for nonvisible, nonpalpable intrapulmonary nodules.
\end{abstract}

Key words: video-assisted thoracoscopic surgery, early-stage lung cancer, near-infrared ray, indocyanine green.

\section{Introduction}

Lung cancer is the leading cause of cancer death in the world [1]. The frequency of detection of ground-glass nodules and small pulmonary metastases has increased due to advances in clinical imaging/staging modalities [2, 3]. A recent clinical trial demonstrated the efficacy of low-dose comnputed tomography (CT) screening for high-risk individuals, potentially increasing the expected detection rate of early-stage lung cancer [4]. Early-stage lung cancer had a chance for less invasive surgery, and achieved a higher survival. Due to the minimal postoperative morbidity and mortality along with less pain and

Address for correspondence

Liang Zhong, Department of Thoracic Surgery, Gansu Provincial Hospital, Lanzhou, China, e-mail: zhonglianggsh@sina.com 
a better quality of life than with an open thoracotomy, video-assisted thoracoscopic surgery (VATS) has been known to be ideal for resection of small nodules $[5,6]$. During VATS, localizing the small-sized pulmonary nodules is challenging when no changes appear on visceral pleura [7]. Inadequate nodule localization may lead to prolonged operative time while searching for the nodule or result in conversion to open thoracotomy $[7,8]$.

With the current shift toward minimally invasive surgery, intraoperative localization of small lung nodules has become a critical challenge. Preoperative localization techniques have been introduced as a method of improving the success rates of VATS and preventing unwanted thoracotomy [7]. Recently developed fluorescence-based optical imaging methods are being used for navigation in various fields of surgery [2]. Indocyanine green (ICG) is used for evaluation of liver function, cardiac output, and so on [9]. The emission of fluorescence by ICG can be detected even within deeply located structures because it is transmitted through tissue [10, 11]; therefore, ICG-based optical imaging can provide minimally invasive imaging of vessels and lymphatic ducts deep inside organs, and real-time imaging during surgery. ICG-based imaging has been recently used in the field of thoracic surgery for early-stage lung tumor.

\section{Aim}

The purpose of the current study is to assess the clinical effectiveness of VATS wedge resection in early-stage lung cancer by ICG for intraoperative tumor mapping.

\section{Material and methods}

This study was prospectively performed with data collected from patients who had undergone VATS wedge resection for early-stage lung cancer at our hospital between March 2016 and August 2019. The inclusion criteria for our study were patients with undiagnosed peripheral nodules that were smaller than $2 \mathrm{~cm}$ scheduled for CT-guided microcoil placement followed by VATS wedge resection. Patients with suspicious mediastinum on $\mathrm{CT}$ or positron emission tomography scan underwent preoperative endobronchial ultrasound transbronchial aspiration to confirm that the mediastinal lymph nodes were clear of disease [12]. The exclusion criteria were as follows: Patients were excluded if they were younger than 19 years of age or older than 85 years of age or had undergone lobectomy or segmentectomy, patients with an allergy to iodine, shellfish, cough mixture, Betadine, or other iodine contrast agents; pregnant or potentially pregnant women. This study was conducted in accordance with the amended Declaration of Helsinki. The institutional review boards of our hospital approved the protocol, and written informed consent was obtained from all patients.

The ICG (IC-Green; Akorn, Inc, Lake Forest, III) is a water-soluble anionic, amphiphilic NIR fluorophore with an excitation wavelength of $790 \mathrm{~nm}$, an emission wavelength of $830 \mathrm{~nm}$, and a molecular weight of $774.9 \mathrm{kDa}$. The ICG was diluted to $2.5 \mathrm{mg} / \mathrm{ml}$ using $25 \%$ human serum albumin (ICG : HSA), as albumin has been shown to increase the effective hydrodynamic diameter of ICG almost 6-fold, resulting in increased lymphatic retention [13]. A dose of 100 to $150 \mathrm{ml}$ of ICG was injected into the peritumoral lung parenchyma percutaneously under CT guidance. The ICG dose was selected on the basis of previous studies to exhibit high fluorescence intensity [14]. The ICG fluorescence in the lung tissue was captured by the NIR thoracoscope, which has a $10-\mathrm{mm}$ diameter and a 30-cm length in a straight, rigid scope. The optical system has a 0-degree view angle, and a 70-degree field of view, transmitting both visible light and $808 \pm 5 \mathrm{~nm}$ NIR laser. The laser power is classified as class $3 R$ at the tip of the scope as per the International Electro-Technical Commission IEC60825-1. Pulse duration is $17 \mathrm{~ms}$ in the maximum setting at 20 pulses per second.

After confirmation of a satisfactory microcoil position on CT (Photos $1 \mathrm{~A}, \mathrm{~B}$ ), the patient was transferred to the cone-beam CT table and underwent induction with general anesthesia. After commencing VATS, the NIR thoracoscope was used to localize the nodule with ICG fluorescence. Thoracoscopic instruments were used to clamp the lung to set an imaginary staple line for the intended resection (Photos 1 C, D). X-ray fluoroscopy was then used to confirm the location of the microcoil in relation to the instrument, and surgical staplers were then used to carry out the VATS wedge resection. Finally, the microcoil was localized using intraoperative fluoroscopy as the standard of care, and the lesion was removed together with the lung parenchyma and the entire microcoil. After resection, the specimen was cut along the maximum diameter of the lung tumor, ob- 

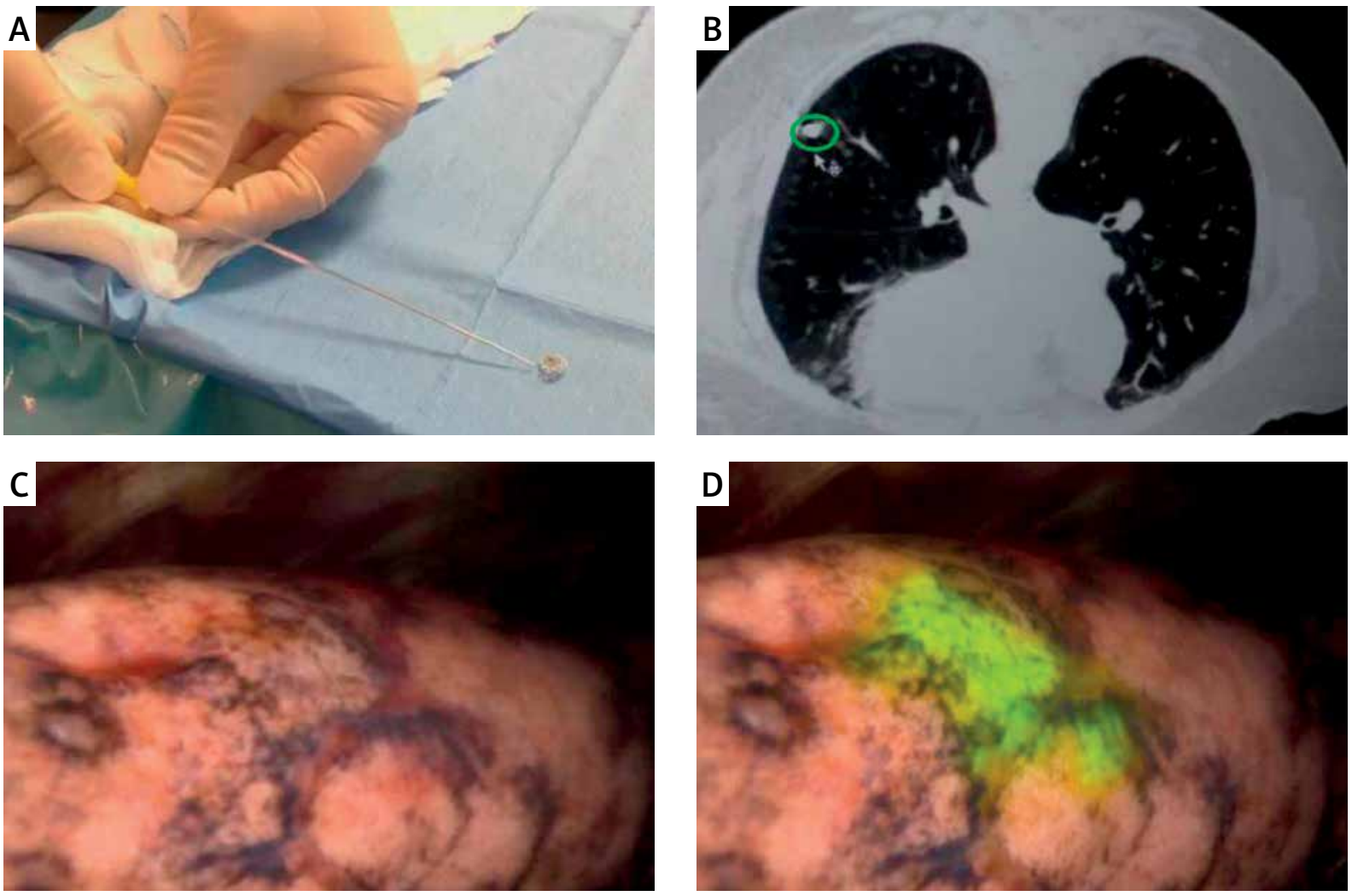

Photo 1. A - The microcoil. B - Lung nodule. C - Intraoperative white light imaging using a NIR thoracoscope. D - Intraoperative white light, and fluorescence merge image using a NIR thoracoscope

served with the NIR thoracoscope, and the surgical margin was inspected macroscopically. If the surgical margin was close to the tumor macroscopically, an additional resection was performed and sent for pathologic evaluation. All tumors were reviewed by specialized lung pathologists.

\section{Results}

Between March 2016 and August 2019, 30 patients with small peripheral nodules suspicious for malignancy underwent CT-guided placement of a microcoil and injection of ICG followed by NIR image-guided VATS resection. All operations described in this trial were wedge resections.

The patient characteristics are described in Table I. There were 20 male and 10 female patients, and their median age was 62 years (range: $32-75$ years). The median forced expiratory volume in $1 \mathrm{~s}$ $\left(\mathrm{FEV}_{1}\right)$ was 2.58 I (range: $\left.1.34-4.65 \%\right)$, and the median predictive diffusing capacity for carbon monoxide (\%DLCO) was 90.5 (range: 57.2-160.2\%). Thirty cases were primary lung cancers, and none were metastatic lung tumors. The tumor data are listed in Table II. The tumors were mainly in the right upper lobe $(n=10,33.33 \%)$ and left upper lobe $(n=11$,

Table I. Baseline characteristics of patients

\begin{tabular}{|lc|}
\hline Variable & Value \\
\hline Age, median (range) [years] & $62(32-75)$ \\
\hline $\begin{array}{l}\text { Gender, } n(\%): \\
\text { Male }\end{array}$ & $20(66.67)$ \\
\hline Female & $10(33.33)$ \\
\hline Primary lung cancer, $n(\%)$ & $30(100)$ \\
\hline $\begin{array}{l}\text { Smoking history, } n(\%): \\
\text { Current and ever }\end{array}$ & $18(60.00)$ \\
\hline $\begin{array}{l}\text { Never } \\
\text { Pulmonary function: }\end{array}$ & $12(40.00)$ \\
\hline FEV ${ }_{1}[1]$, mean (range) & $90.5(57.2-160.2)$ \\
\hline
\end{tabular}

$F E V_{1}$-forced expiratory volume in $1 \mathrm{~s}, \% D L C O$ - predictive diffusing capacity for carbon monoxide. 
Table II. Tumor data of patients

\begin{tabular}{|lc|}
\hline Variable & Value \\
\hline CT nodule size, median (range) [cm] & $1.3(0.6-1.9)$ \\
\hline $\begin{array}{l}\text { CT nodule depth from surface, median } \\
\text { (range) [cm] }\end{array}$ & $1.7(0.5-3.8)$ \\
\hline \begin{tabular}{l} 
Nodule location, $n(\%):$ \\
\hline Right upper lobe
\end{tabular} & $10(33.33)$ \\
\hline \begin{tabular}{l} 
Right middle lobe \\
\hline Right lower lobe
\end{tabular} & $2(6.67)$ \\
\hline $\begin{array}{l}\text { Left upper lobe } \\
\text { Left lower lobe }\end{array}$ & $11(36.67)$ \\
\hline CT image findings, $n(\%):$ & $21(70.00)$ \\
\hline $\begin{array}{l}\text { Pure GGN } \\
\text { Solid nodule }\end{array}$ \\
\hline
\end{tabular}

CT - computed tomography, GGN - ground-glass nodule.

$36.67 \%$ ). On $\mathrm{CT}$, there were 9 solid nodules (< $50 \%$ consolidation/tumor $(\mathrm{C} / \mathrm{T})$ ratio $\leq 100 \%)$, and 21 pure ground-glass nodules $(\mathrm{C} / \mathrm{T}$ ratio $=0 \%)$. The median CT tumor size was $1.3 \mathrm{~cm}$ (range: $0.6-1.9 \mathrm{~cm}$ ), and the pathologic tumor size of 30 cases was $1.2 \mathrm{~cm}$ (range: $0.5-1.9 \mathrm{~cm}$ ). The median distance from the pleural surface to the nodal surface was $1.7 \mathrm{~cm}$ (range: $0.5-3.8 \mathrm{~cm}$ ).

The surgical outcomes of patients are summarized in Table III. The median CT-guided intervention time was 25 min (range: 19-49 min), and VATS wedge resection procedural time was 50 min (range: 42-80 min). The median intraoperative blood loss was $20 \mathrm{ml}$ (range: 10-50 ml). ICG was successfully injected in all patients. During surgery, ICG fluorescence was clearly identified in all cases. In 30 cases, the NIR thoracoscope was solely used to successfully localize and resect the nodule without the need of fluoroscopy. After wedge resection, we confirmed NIR fluorescence in the resected specimen. In all cases, the target nodule was successfully removed with negative surgical margins, and ICG fluorescence was clearly detected in the resected specimen. There were no adverse effects related to ICG injection. We performed intraoperative lymph node sampling in 5 cases to confirm negative lymph node metastasis. There were no perioperative or postoperative complications. All patients were discharged from the hospital without any complication related to the procedure.
Table III. Surgical outcomes of patients

\begin{tabular}{|c|c|}
\hline Variable & Value \\
\hline Operation time, median (range) [min] & $50(42-80)$ \\
\hline $\begin{array}{l}\text { CT-guided intervention time, median (range) } \\
\text { [min] }\end{array}$ & $25(19-49)$ \\
\hline Intraoperative blood loss, median (range) [ml] & $20(10-50)$ \\
\hline Conversion to thoracotomy, $n(\%)$ & $0(0.00)$ \\
\hline ICGF, $n(\%)$ & $10(33.33)$ \\
\hline Clear, $n(\%)$ & $30(100.00)$ \\
\hline Unclear, $n(\%)$ & $0(0.00)$ \\
\hline Non-detect, $n(\%)$ & $0(0.00)$ \\
\hline \multicolumn{2}{|l|}{ Postoperative complication: } \\
\hline \multicolumn{2}{|l|}{ Pneumonia: } \\
\hline IP/AE, $n(\%)$ & $21(70.00)$ \\
\hline \multicolumn{2}{|l|}{ Subcutaneous hematoma: } \\
\hline Chest drainage, median (range) [days] & $2(1-7)$ \\
\hline Hospitalization, median (range) [days] & $6(4-10)$ \\
\hline \multicolumn{2}{|l|}{ Pathologic diagnosis, $n$ (\%): } \\
\hline Ad (include AIS and MIA) & $21(70.00)$ \\
\hline $\mathrm{Sq}$ & $3(10.00)$ \\
\hline Ad-sq & $3(10.00)$ \\
\hline SCLC & $3(10.00)$ \\
\hline Pathologic tumor size, median (range) $[\mathrm{cm}]$ & $1.2(0.5-1.9)$ \\
\hline \multicolumn{2}{|l|}{ Surgical margin, $n(\%)$ : } \\
\hline$\geq$ Tumor size & $30(100.00)$ \\
\hline$<$ Tumor size & $0(0.00)$ \\
\hline $\begin{array}{l}\text { Pathologic closest resection margin, median } \\
\text { (range) }[\mathrm{cm}]\end{array}$ & $1.0(0.7-1.9)$ \\
\hline
\end{tabular}

ICGF - indocyanine green fluorescence, IP/AE - acute postoperative exacerbation of interstitial pneumonia, Ad - adenocarcinoma, AIS - adenocarcinoma in situ, MIA - minimally invasive adenocarcinoma, Sq - squamous cell carcinoma, SCLC - small cell lung cancer.

The final diagnoses of the 30 cases included 21 adenocarcinomas (70\%), 3 squamous cell carcinomas (10\%), 3 adenocarcinoma squamous cell carcinomas (10\%) and 3 small cell carcinomas (10\%). Among the 21 primary pulmonary adenocarcinoma cases, 15 were adenocarcinomas in situ, and 6 were minimally invasive adenocarcinomas. The surgical margins of all wedge resection specimens were negative microscopically without additional resection of lung parenchyma. 


\section{Discussion}

This is the first reported clinical trial to demonstrate the safety and feasibility of CT-guided peritumoral NIR marking of lung lesions and mapping of tumor in China. In our clinical trial, 30 patients with solitary pulmonary nodule underwent VATS wedge resection after ICG injection. The NIR signal detected $100 \%$ of these tumors without complications. On the basis of our study, CT-guided percutaneous ICG injection with intraoperative NIR localization of small nodules was a safe and feasible procedure during minimally invasive surgery. It offers surgeons the ease of localization through direct ICG fluorescence imaging without the use of fluoroscopy and appears to be equivalent in localization accuracy to preoperative microcoil placement for nonvisible, nonpalpable intrapulmonary nodules.

Under the current NCCN guidelines, patients with small, peripheral semi-solid, or slow-growing lesions may not require a lobectomy (although an anatomic resection is still preferred) [15], and some studies indicate favorable long-term outcomes with limited resection $[16,17]$. These small, ill-defined lesions may be difficult to localize intraoperatively; however, with this technique, they can easily be identified and staged in a directed fashion and in a single operative setting. This is important for patients undergoing wedge resection. In the current study, we used new NIR thoracoscope technology with the ability to simultaneously display and overlay a fluorescence image and a white light image during VATS (PINPOINT Endoscopic Fluorescence Imaging; Novadaq Technologies Inc, Mississauga, Ontario, Canada). NIR imaging using ICG fluorescence has several unique advantages compared with other techniques. Furthermore, this technique for targeted nonvisible, nonpalpable intrapulmonary nodule mapping may offer an advantage for poor surgical candidates who cannot tolerate a large anatomic resection but would receive better staging than a patient undergoing focused definitive radiation such as stereotactic body radiation therapy.

The ICG fluorescence penetrating the lung tissue was successfully captured by the NIR thoracoscope in 30 cases and was detected regardless of the color of the lung surface. Unlike color dye detection by a white light endoscope, the specific wavelength of ICG fluorescence is always detectable regardless of any changes in color or texture of the visceral pleura, which may occur as the result of anthracosis or other underlying pulmonary diseases. ICG fluorescence imaging is easy to understand, real-time, and intuitive for the observer. Furthermore, compared with other fiducial markers, ICG is safe and does not necessarily have to be removed from lung tissue.

We acknowledge that the present method has limitations and thus room for technical improvement. On examination of the resected specimen, ICG fluorescence was clearly detected near the microcoil and the tumor. As demonstrated in these cases, the depth of the lesion and complete deflation of the lung are important factors for optimal ICG fluorescence imaging; thus, these factors should be taken into account when selecting patients for ICG localization. The next study in preparation is to perform ICG localization without the use of a microcoil. Different injection approaches are under consideration. Limitations of the current prospective study include a small sample size, and limited histologic and radiographic variability in tumor type. In addition, long-term recurrence and survival outcomes are necessary in larger, multicenter trials to determine whether cases identified as NO based on SLN status behave as true node-negative cases and exhibit better overall survival than patients deemed node-negative by standard hematoxylin and eosin analysis of routine LAD specimens.

\section{Conclusions}

We have described a new multimodality approach for image-guided localization and minimally invasive resection of small pulmonary nodules using a NIR thoracoscope and ICG fluorescence. This is a safe and feasible minimally invasive approach for localization and resection of small and ground-glass nodules. It is a complementary technique to CT-guided microcoil localization.

\section{Conflict of interest}

The authors declare no conflict of interest.

\section{References}

1. Canadian Cancer Society. Lung cancer statistics. Available at: http://www.cancer.ca/en/cancer-information/cancer-type/ lung/statistics/?region=on. Accessed May 24, 2016.

2. Schaafsma BE, Mieog JS, Hutteman M, et al. The use of indocyanine green as a near-infrared fluorescent contrast agent for image-guided oncologic surgery. J Surg Oncol 2011; 104: 323-32. 
3. Gioux S, Choi HS, Frangioni JV. Image-guided surgery using invisible near-infrared light: fundamentals of clinical translation. Mol Imaging 2010; 9: 237-55.

4. Aberle DR, Adams AM, Berg CD, et al. Reduced lung-cancer mortality with low-dose computed tomographic screening. N Engl J Med 2011; 365: 395-409.

5. Bendixen M, Jorgensen OD, Kronborg C, et al. Postoperative pain and quality of life after lobectomy via video-assisted thoracoscopic surgery or anterolateral thoracotomy for early stage lung cancer: a randomised controlled trial. Lancet Oncol 2016; 17: 836-44.

6. Vannucci F, Gonzalez-Rivas D. Is VATS lobectomy standard of care for operable non-small cell lung cancer? Lung Cancer 2016; 100: 114-9.

7. Saito H, Minamiya Y, Matsuzaki I, et al. Indication for preoperative localization of small peripheral pulmonary nodules in thoracoscopic surgery. J Thorac Cardiovasc Surg 2002; 124: 1198-202.

8. Powell TI, Jangra D, Clifton JC, et al. Peripheral lung nodules: fluoroscopically guided video-assisted thoracoscopic resection after computed tomography-guided localization using platinum microcoils. Ann Surg 2004; 240: 481-9.

9. Ogawa M, Kosaka N, Choyke PL, et al. In vivo molecular imaging of cancer with a quenching near-infrared fluorescent probe using conjugates of monoclonal antibodies and indocyanine green. Cancer Res 2009; 69: 1268-72.

10. Yamamoto M, Orihashi K, Nishimori H, et al. Efficacy of intraoperative HyperEye Medical System angiography for coronary artery bypass grafting. Surg Today 2015; 45: 966-72.

11. Handa T, Katare RG, Nishimori H, et al. New device for intraoperative graft assessment: HyperEye charge-coupled device camera system. Gen Thorac Cardiovasc Surg 2010; 58: 68-77.

12. Yasufuku K, Pierre A, Darling G, et al. A prospective controlled trial of endobronchial ultrasound-guided transbronchial needle aspiration compared with mediastinoscopy for mediastinal lymph node staging of lung cancer. J Thorac Cardiovasc Surg 2011; 142: 1393-400.

13. Gilmore DM, Khullar OV, Colson YL. Developing intrathoracic sentinel lymph node mapping with near infrared fluorescent imaging in non-small cell lung cancer. J Thorac Cardiovasc Surg 2012; 144: S80-4.

14. Anayama T, Qiu J, Chan H, et al. Localization of pulmonary nodules using navigation bronchoscope and a near-infrared fluorescence thoracoscope. Ann Thorac Surg 2015; 99: 224-30.

15. Ettinger DS, Wood DE, Akerley W, et al. NCCN guidelines in sights: non-small cell lung cancer, version 4.2016. J Natl Compr Canc Netw 2016; 14: 255-64.

16. Altorki NK, Yip R, Hanaoka T, et al. Sublobar resection is equivalent to lobectomy for clinical stage $1 \mathrm{~A}$ lung cancer in solid nod ules. J Thorac Cardiovasc Surg 2014; 147: 754-62.

17. Schuchert MJ, Kilic A, Pennathur A, et al. Oncologic outcomes after surgical resection of subcentimeter non-small cell lung cancer. Ann Thorac Surg 2011; 91: 1687-8.

Received: 5.10.2019, accepted: 14.10.2019. 